MATHEMATICS OF COMPUTATION

Volume 69, Number 232, Pages 1319-1339

S 0025-5718(00)01236-9

Article electronically published on April 12, 2000

\title{
LOWER BOUNDS FOR NONOVERLAPPING DOMAIN DECOMPOSITION PRECONDITIONERS IN TWO DIMENSIONS
}

\author{
SUSANNE C. BRENNER AND LI-YENG SUNG
}

\begin{abstract}
Lower bounds for the condition numbers of the preconditioned systems are obtained for the Bramble-Pasciak-Schatz substructuring preconditioner and the Neumann-Neumann preconditioner in two dimensions. They show that the known upper bounds are sharp.
\end{abstract}

\section{INTRODUCTION}

Domain decomposition methods (cf. [5, 16], [22]) provide parallel algorithms for the numerical solution of partial differential equations. One of the indicators of the efficiency of a domain decomposition preconditioner is the rate of growth of the condition number of the preconditioned system, which usually comes in the form of an upper bound. In this paper we will establish lower bounds for two wellknown nonoverlapping domain decomposition preconditioners in two dimensions: the substructuring preconditioner of Bramble, Pasciak and Schatz (cf. 3]) and the Neumann-Neumann preconditioner (cf. [9, 10, [16, 11 and the references therein). Our results show that the known upper bounds for these algorithms are sharp.

We will establish the lower bounds within the framework of additive Schwarz preconditioners, which can be summarized as follows, where all vector spaces are real and have finite dimensions.

Let $V$ be a vector space, $V^{\prime}$ be the dual space of $V$, and $\langle\cdot, \cdot\rangle$ be the canonical bilinear form on $V^{\prime} \times V$, i.e., $\langle\alpha, v\rangle=\alpha(v) \forall \alpha \in V^{\prime}, v \in V$. We say that a linear operator $A: V \longrightarrow V^{\prime}$ is symmetric positive definite (SPD) if $\left\langle A v_{1}, v_{2}\right\rangle=$ $\left\langle A v_{2}, v_{1}\right\rangle \forall v_{1}, v_{2} \in V$ and $\langle A v, v\rangle>0 \forall 0 \neq v \in V$.

Let $V_{j}, 0 \leq j \leq J$, be vector spaces and $B_{j}: V_{j} \longrightarrow V_{j}^{\prime}$ be linear SPD operators. The additive Schwarz preconditioner $B: V^{\prime} \longrightarrow V$ for the linear SPD operator $A: V \longrightarrow V^{\prime}$ is defined in terms of the $B_{j}$ 's by the formula

$$
B=\sum_{j=0}^{J} I_{j} B_{j}^{-1} I_{j}^{t},
$$

Received by the editor May 22, 1998 .

1991 Mathematics Subject Classification. Primary 65N55, 65N30.

Key words and phrases. Lower bounds, nonoverlapping domain decomposition preconditioners, Bramble-Pasciak-Schatz, Neumann-Neumann, two dimensions.

The work of the first author was supported in part by the National Science Foundation under Grant No. DMS-96-00133. 
where $I_{j}: V_{j} \longrightarrow V$ is a linear operator that connects $V_{j}$ to $V$, and the operator $I_{j}^{t}: V^{\prime} \longrightarrow V_{j}^{\prime}$ is defined by $\left\langle I_{j}^{t} \alpha, v\right\rangle=\left\langle\alpha, I_{j} v\right\rangle \forall \alpha \in V^{\prime}, v \in V_{j}$.

The operator $B A: V \longrightarrow V$ is clearly symmetric positive semidefinite with respect to the inner product $\langle A \cdot, \cdot\rangle$. Under the condition

$$
V=\sum_{j=0}^{J} I_{j} V_{j},
$$

the operator $B$ is invertible and $B A$ is symmetric positive definite. The eigenvalues of $B A$ are therefore positive, and we have the following characterizations (cf. [19, [17], 20], 21], 8], 22], 24], 25], [12]) for the minimum and maximum eigenvalues of $B A$ :

$$
\begin{aligned}
& \lambda_{\min }(B A)=\min _{0 \neq v \in V} \frac{\langle A v, v\rangle}{\min _{\substack{v=\sum_{j=0}^{J} I_{j} v_{j} \\
v_{j} \in V_{j}}} \sum_{j=0}^{J}\left\langle B_{j} v_{j}, v_{j}\right\rangle}, \\
& \lambda_{\max }(B A)=\max _{0 \neq v \in V} \frac{\langle A v, v\rangle}{\min _{\substack{v=\sum_{j=0}^{J} I_{j} v_{j} \\
v_{j} \in V_{j}}} \sum_{j=0}^{J}\left\langle B_{j} v_{j}, v_{j}\right\rangle} .
\end{aligned}
$$

In order to obtain a lower bound for the condition number $\kappa(B A)=\frac{\lambda_{\max }(B A)}{\lambda_{\min }(B A)}$, we need to find a lower bound $\mathcal{L}$ for $\lambda_{\max }(B A)$ and an upper bound $\mathcal{U}$ for $\lambda_{\min }(B A)$. In view of (1.3) and (1.4), the strategy for establishing the lower bound $\mathcal{L}$ of $\lambda_{\max }(B A)$ is to find one $0 \neq v_{*} \in V$ and one decomposition $v_{*}=\sum_{j=0}^{J} I_{j} v_{j}$ for which we have $\left\langle A v_{*}, v_{*}\right\rangle \geq \mathcal{L} \sum_{j=0}^{J}\left\langle B_{j} v_{j}, v_{j}\right\rangle$, and the strategy for establishing the upper bound $\mathcal{U}$ of $\lambda_{\min }(B A)$ is to find one $0 \neq v_{\dagger} \in V$ such that $\left\langle A v_{\dagger}, v_{\dagger}\right\rangle \leq \mathcal{U} \sum_{j=0}^{J}\left\langle B_{j} v_{j}, v_{j}\right\rangle$ holds for any decomposition $v_{\dagger}=\sum_{j=0}^{J} I_{j} v_{j}$.

Based on these strategies we will show that, for a second order model finite element problem, the condition number of the preconditioned system is bounded below by $c[1+\ln (H / h)]^{2}$ for both the BPS preconditioner and the Neumann-Neumann preconditioner, where $H$ represents the diameter of a typical subdomain, $h$ is the mesh size of the triangulation and the constant $c$ is independent of $H, h$ and the number of subdomains.

The rest of the paper is organized as follows. The descriptions of the model finite element problem and the preconditioners are given in Section 2. The constructions of the functions $v_{*}$ and $v_{\dagger}$ in the strategies stated above are based on the constructions of special one dimensional piecewise linear functions, which are carried out in Section 3. The lower bounds for the BPS preconditioner and the Neumann-Neumann preconditioner are then established in Sections 4 and 5, respectively. Section 6 contains the proofs of two technical lemmas from Section 3.

For the convenience of the readers, we state here the definitions of the Sobolev norms and seminorms that are used throughout this paper. 
Let $\Omega$ be a bounded open subset of $\mathbb{R}^{n}$ and $|\Omega|$ be the $n$-dimensional Lebesgue measure of $\Omega$. We define

$$
\begin{aligned}
\|v\|_{L_{2}(\Omega)}^{2} & =\frac{1}{|\Omega|} \int_{\Omega}|v|^{2} d x, \\
|v|_{H^{1}(\Omega)}^{2} & =\frac{1}{|\Omega|^{1-(2 / n)}} \int_{\Omega}|\nabla v|^{2} d x, \\
\|v\|_{H^{1}(\Omega)}^{2} & =\|v\|_{L_{2}(\Omega)}^{2}+|v|_{H^{1}(\Omega)}^{2} .
\end{aligned}
$$

For a bounded open interval $I \subseteq \mathbb{R}$, we define

$$
\begin{aligned}
|v|_{H^{1 / 2}(I)}^{2} & =\int_{I} \int_{I} \frac{|v(x)-v(y)|^{2}}{|x-y|^{2}} d x d y, \\
\|v\|_{H^{1 / 2}(I)}^{2} & =\|v\|_{L_{2}(I)}^{2}+|v|_{H^{1 / 2}(I)}^{2},
\end{aligned}
$$

and for a bounded open set $\Omega \subseteq \mathbb{R}^{2}$ with a $C^{0,1}$ boundary, we define

$$
\begin{aligned}
\|v\|_{L_{2}(\partial \Omega)}^{2} & =\frac{1}{|\partial \Omega|} \int_{\partial \Omega}|v|^{2} d s, \\
|v|_{H^{1 / 2}(\partial \Omega)}^{2} & =\int_{\partial \Omega} \int_{\partial \Omega} \frac{|v(x)-v(y)|^{2}}{|x-y|^{2}} d s(x) d s(y), \\
\|v\|_{H^{1 / 2}(\partial \Omega)}^{2} & =\|v\|_{L_{2}(\partial \Omega)}^{2}+|v|_{H^{1 / 2}(\partial \Omega)}^{2},
\end{aligned}
$$

where $|\partial \Omega|$ is the arc-length of $\partial \Omega$, and $d s$ is the differential of the arc-length.

Note that the norms and seminorms defined by $(1.5)-(1.12)$ are invariant under translation and scaling. Also, the inner products $(\cdot, \cdot)_{L_{2}},(\cdot, \cdot)_{H^{1}}$ and $(\cdot, \cdot)_{H^{1 / 2}}$ are defined by the polarization identities of the corresponding norms $\|\cdot\|_{L_{2}},\|\cdot\|_{H^{1}}$ and $\|\cdot\|_{H^{1 / 2}}$.

\section{The MOdel PRoblem AND the PRECONDItioners}

Since our goal is to show that the known condition number estimates for the BPS preconditioner and the Neumann-Neumann preconditioner are sharp, it suffices to consider the simplest model problem.

Let $\Omega=(-1,1) \times(-1,1)$. The variational formulation for the Poisson equation on $\Omega$ with homogeneous Dirichlet boundary condition follows.

Find $u \in H_{0}^{1}(\Omega)$ such that

$$
a(u, v)=\int_{\Omega} f v d x \quad \forall v \in H_{0}^{1}(\Omega),
$$

where $f \in L_{2}(\Omega)$, and the variational form $a(\cdot, \cdot)$ is defined by

$$
a\left(v_{1}, v_{2}\right)=\int_{\Omega} \nabla v_{1} \cdot \nabla v_{2} d x \quad \forall v_{1}, v_{2} \in H_{0}^{1}(\Omega) .
$$

Anticipating the use of nonoverlapping preconditioners, we construct a triangulation of $\Omega$ in the following way. Let $\Omega$ be divided into $J=2^{2 k}$ nonoverlapping squares $\Omega_{1}, \ldots, \Omega_{J}$ (cf. Figure 1 where $k=2$ ). By adding a diagonal to each $\Omega_{j}$ we obtain a triangulation $\mathcal{T}_{H}$ of $\Omega$ (cf. Figure 2). Then we perform a regular subdivision of $\mathcal{T}_{H}$ to obtain the triangulation $\mathcal{T}_{h}$ (cf. Figure 3). Here $H$ and $h$ are the lengths of the horizontal edges in $\mathcal{T}_{H}$ and $\mathcal{T}_{h}$, respectively. 


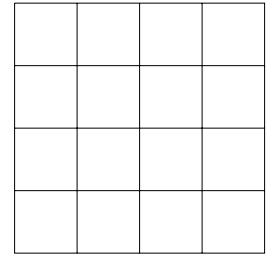

Figure 1.

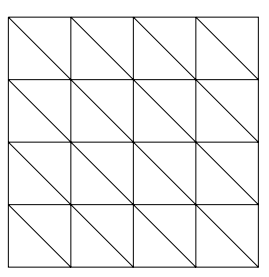

FiguRE 2.

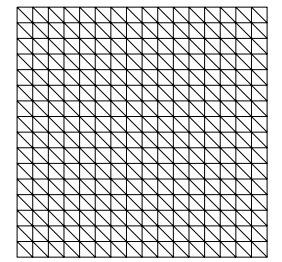

FIGURE 3.

Let $V_{h} \subseteq H_{0}^{1}(\Omega)$ be the $P_{1}$ finite element space associated with $\mathcal{T}_{h}$. The discretization of (2.1) is to find $u_{h} \in V_{h}$ such that

$$
a\left(u_{h}, v\right)=\int_{\Omega} f v d x \quad \forall v \in V_{h} .
$$

In a nonoverlapping domain decomposition method we split the unknown $u_{h}$ in (2.3) into two components with respect to the skeleton $\Gamma=\bigcup_{j=1}^{J}\left(\partial \Omega_{j} \backslash \partial \Omega\right)$ as follows. Let $V_{h}(\Omega \backslash \Gamma)=\left\{v \in V_{h}: v\right.$ vanishes on $\left.\Gamma\right\}$ and $V_{h}(\Gamma) \subseteq V_{h}$ be the $a(\cdot, \cdot)$ orthogonal complement of $V_{h}(\Omega \backslash \Gamma)$, i.e.,

$$
V_{h}(\Gamma)=\left\{v \in V_{h}: a(v, w)=0 \quad \forall w \in V_{h}(\Omega \backslash \Gamma)\right\} .
$$

The functions in $V_{h}(\Gamma)$ are known as discrete harmonic functions and they are completely determined by their nodal values along $\Gamma$. We can write $u_{h}=\dot{u}_{h}+\bar{u}_{h}$, where $\dot{u}_{h} \in V_{h}(\Omega \backslash \Gamma)$ and $\bar{u}_{h} \in V_{h}(\Gamma)$. The two components $\dot{u}_{h}$ and $\bar{u}_{h}$ are determined by

$$
\begin{array}{ll}
a\left(\dot{u}_{h}, v\right)=\int_{\Omega} f v d x & \forall v \in V_{h}(\Omega \backslash \Gamma), \\
a\left(\bar{u}_{h}, v\right)=\int_{\Omega} f v d x & \forall v \in V_{h}(\Gamma) .
\end{array}
$$

Since $\dot{u}_{h}$ can be obtained from (2.5) by solving in parallel a Dirichlet problem in each subdomain, the goal of a nonoverlapping domain decomposition method is to provide a good preconditioner for the system (2.6) so that it can be solved efficiently by, for example, the preconditioned conjugate gradient method.

Let $S_{h}: V_{h}(\Gamma) \longrightarrow V_{h}(\Gamma)^{\prime}$ be defined by

$$
\left\langle S_{h} v_{1}, v_{2}\right\rangle=a\left(v_{1}, v_{2}\right) \quad \forall v_{1}, v_{2} \in V_{h}(\Gamma) .
$$

We can write $(2.6)$ as $S_{h} \bar{u}_{h}=\bar{f}_{h}$, where $\bar{f}_{h} \in\left[V_{h}(\Gamma)\right]^{\prime}$ is defined by $\left\langle\bar{f}_{h}, v\right\rangle=$ $\int_{\Omega} f v d x$. The operator $S_{h}$, known as the Schur complement operator, is then the one that we want to precondition.

Below we will describe the BPS preconditioner and the Neumann-Neumann preconditioner for $S_{h}$. In both methods we use the coarse grid space $V_{H} \subseteq H_{0}^{1}(\Omega)$, which is the $P_{1}$ finite element space associated with the triangulation $\mathcal{T}_{H}$. The space $V_{H}$ is connected to $V_{h}(\Gamma)$ by the operator $I_{H}: V_{H} \longrightarrow V_{h}(\Gamma)$ defined by

$$
\left.\left(I_{H} v\right)\right|_{\Gamma}=\left.v\right|_{\Gamma} \quad \forall v \in V_{H}
$$

and the linear SPD operator $A_{H}: V_{H} \longrightarrow V_{H}^{\prime}$ is defined by

$$
\left\langle A_{H} v_{1}, v_{2}\right\rangle=a\left(v_{1}, v_{2}\right) \quad \forall v_{1}, v_{2} \in V_{H} .
$$


BPS preconditioner. Let $E_{\ell}, 1 \leq \ell \leq L$, be the common edge of neighboring subdomains without the endpoints, and let the edge space $V_{h}\left(E_{\ell}\right)$ be defined by

$$
V_{h}\left(E_{\ell}\right)=\left\{v \in V_{h}(\Gamma): v=0 \text { on } \Gamma \backslash E_{\ell}\right\} .
$$

Each $V_{h}\left(E_{\ell}\right)$ is connected to $V_{h}(\Gamma)$ by the natural injection $I_{\ell}: V_{h}\left(E_{\ell}\right) \longrightarrow V_{h}(\Gamma)$, and there is a linear SPD operator $S_{\ell}: V_{h}\left(E_{\ell}\right) \longrightarrow V_{h}\left(E_{\ell}\right)^{\prime}$ defined by

$$
\left\langle S_{\ell} v_{1}, v_{2}\right\rangle=a\left(v_{1}, v_{2}\right) \quad \forall v_{1}, v_{2} \in V_{h}\left(E_{\ell}\right) .
$$

The BPS preconditioner $B_{B P S}: V_{h}(\Gamma)^{\prime} \longrightarrow V_{h}(\Gamma)$ is given by

$$
B_{B P S}=I_{H} A_{H}^{-1} I_{H}^{t}+\sum_{\ell=1}^{L} I_{\ell} S_{\ell}^{-1} I_{\ell}^{t} .
$$

It is clear that (1.2) is satisfied. In fact, we have the stronger condition

$$
V_{h}(\Gamma)=I_{H} V_{H} \oplus V_{h}\left(E_{1}\right) \oplus \cdots \oplus V_{h}\left(E_{L}\right) .
$$

The following condition number estimate (cf. 3]) holds:

$$
\kappa\left(B_{B P S} S_{h}\right) \leq C\left(1+\ln \frac{H}{h}\right)^{2},
$$

where the positive constant $C$ is independent of $H, h$ and $J$.

Remark 2.1. In the original BPS algorithm (cf. [3]) the exact solves $S_{j}^{-1}$ are replaced by spectrally equivalent interface preconditioners that are easier to compute. But for our purpose we may as well use exact solves.

Remark 2.2. There is numerical evidence (cf. [3]) that the estimate (2.14) is sharp. A mathematical proof will be given in Section 4 .

Neumann-Neumann preconditioner. Let $V_{j}, 1 \leq j \leq J$, be the restriction of $V_{h}$ to $\Omega_{j}$, i.e., $V_{j}$ is the $P_{1}$ finite element space on $\Omega_{j}$ associated with the triangulation $\mathcal{T}_{h}$ whose members vanish on $\partial \Omega \cap \partial \Omega_{j}$. The skeleton $\partial \Omega_{j} \backslash \partial \Omega$ of $\Omega_{j}$ is denoted by $\Gamma_{j}$.

The SPD bilinear form $\hat{a}_{j}(\cdot, \cdot)$ is defined by

$$
\hat{a}_{j}\left(v_{1}, v_{2}\right)=\int_{\Omega_{j}} \nabla v_{1} \cdot \nabla v_{2} d x+H^{-2} \int_{\Omega_{j}} v_{1} v_{2} d x \quad \forall v_{1}, v_{2} \in H^{1}\left(\Omega_{j}\right) .
$$

Let $V_{h}\left(\Omega_{j}\right)=\left\{v \in V_{j}: v\right.$ vanishes on $\left.\partial \Omega_{j}\right\}$ and $V_{h}\left(\Gamma_{j}\right) \subseteq V_{j}$ be the $\hat{a}_{j}(\cdot, \cdot)$ orthogonal complement of $V_{h}\left(\Omega_{j}\right)$, i.e.,

$$
V_{h}\left(\Gamma_{j}\right)=\left\{v \in V_{j}: \hat{a}_{j}(v, w)=0 \quad \forall w \in V_{h}\left(\Omega_{j}\right)\right\} .
$$

The functions in $V_{h}\left(\Gamma_{j}\right)$ are discrete harmonic with respect to the bilinear form $\hat{a}_{j}(\cdot, \cdot)$, and are determined by their nodal values on $\Gamma_{j}$.

Each $V_{h}\left(\Gamma_{j}\right)$ is connected to $V_{h}(\Gamma)$ by $\hat{I}_{j}: V_{h}\left(\Gamma_{j}\right) \longrightarrow V_{h}(\Gamma)$ defined by

$$
\left(\hat{I}_{j} v\right)(p)= \begin{cases}0 & \text { if the node } p \text { is not on } \Gamma_{j}, \\ v(p) / n(p) & \text { if the node } p \text { is on } \Gamma_{j},\end{cases}
$$

where $n(p)$ is the number of subdomains sharing the node $p$. There is also an SPD linear operator $\hat{S}_{j}: V_{h}\left(\Gamma_{j}\right) \longrightarrow V_{h}\left(\Gamma_{j}\right)^{\prime}$ defined by

$$
\left\langle\hat{S}_{j} v_{1}, v_{2}\right\rangle=\hat{a}_{j}\left(v_{1}, v_{2}\right) \quad \forall v_{1}, v_{2} \in V_{h}\left(\Gamma_{j}\right) .
$$


The Neumann-Neumann preconditioner $B_{N N}: V_{h}(\Gamma) \longrightarrow V_{h}(\Gamma)^{\prime}$ is given by

$$
B_{N N}=I_{H} A_{H}^{-1} I_{H}^{t}+\sum_{j=1}^{J} \hat{I}_{j} \hat{S}_{j}^{-1} \hat{I}_{j}^{t}
$$

It is easy to check that (1.2) holds.

The following condition number estimate (cf. [9], [10, [11]) holds:

$$
\kappa\left(B_{N N} S_{h}\right) \leq C\left(1+\ln \frac{H}{h}\right)^{2},
$$

where the positive constant $C$ is independent of $H, h$ and $J$.

Remark 2.3. For a subdomain $\Omega_{j}$ that has at least one side on $\partial \Omega$, we can define $\hat{a}_{j}(\cdot, \cdot)$ by

$$
\hat{a}_{j}\left(v_{1}, v_{2}\right)=\int_{\Omega_{j}} \nabla v_{1} \cdot \nabla v_{2} d x \quad \forall v_{1}, v_{2} \in H^{1}\left(\Omega_{j}\right),
$$

and then define the space $V_{h}\left(\Gamma_{j}\right)$ accordingly. The results in Section 5 for the Neumann-Neumann preconditioner also hold for this choice of $\hat{a}_{j}(\cdot, \cdot)$.

Remark 2.4. Numerical results for the Neumann-Neumann preconditioner without a coarse grid space can be found in [15].

For future reference, we collect some well-known facts concerning discrete harmonic functions and the space $H^{1 / 2}$ in the following lemma . The proofs of these facts can be found either in [3], [7], or by straightforward calculations using (1.10)(1.12).

Lemma 2.5. Let $D$ be a square with a uniform triangulation $\mathcal{T}_{h}$, and $\mathcal{V}_{h} \subseteq H^{1}(D)$ be the $P_{1}$ finite element space associated with $\mathcal{T}_{h}$. Suppose that $v \in \mathcal{V}_{h}$ is discrete harmonic with respect to the bilinear form $d(\cdot, \cdot)$ defined by

$$
d\left(v_{1}, v_{2}\right)=\int_{D} \nabla v_{1} \cdot \nabla v_{2} d x
$$

i.e., $d(v, w)=0$ for all $w \in \mathcal{V}_{h}$ which vanishes on $\partial D$. Then we have $|v|_{H^{1}(D)} \approx$ $|v|_{H^{1 / 2}(\partial D)}$. On the other hand, if $v \in \mathcal{V}_{h}$ is discrete harmonic with respect to the bilinear form $\hat{d}(\cdot, \cdot)$ defined by

$$
\hat{d}\left(v_{1}, v_{2}\right)=\int_{D} \nabla v_{1} \cdot \nabla v_{2} d x+\frac{1}{|D|} \int_{D} v_{1} v_{2} d x,
$$

i.e., $\hat{d}(v, w)=0$ for all $w \in \mathcal{V}_{h}$ which vanishes on $\partial D$, then we have $\|v\|_{H^{1}(D)} \approx$ $\|v\|_{H^{1 / 2}(\partial D)}$. Moreover, for any function $v \in H^{1}(D)$ which vanishes on one side of $\partial D$, we have $\|v\|_{H^{1 / 2}(\partial D)} \approx|v|_{H^{1 / 2}(\partial D)}$.

\section{Special one Dimensional pieceWise Linear FunCtions}

Let $(a, b)$ be a finite open interval. The space $H_{00}^{1 / 2}(a, b)$ plays an important role in the theory of nonoverlapping domain decomposition methods in two dimensions, and it is defined by

$$
H_{00}^{1 / 2}(a, b)=\left\{v \in L_{2}(a, b): \tilde{v} \in H^{1 / 2}(\mathbb{R})\right\},
$$


where $\tilde{v}$ is the trivial extension of $v$ to $\mathbb{R}$, i.e., $\tilde{v}(x)=\left\{\begin{array}{ll}v(x) & x \in(a, b) \\ 0 & x \in \mathbb{R} \backslash(a, b)\end{array}\right.$. We define the norm of $v \in H_{00}^{1 / 2}(a, b)$ to be $|\tilde{v}|_{H^{1 / 2}(\mathbb{R})}$, i.e.,

$$
\begin{aligned}
|v|_{H_{00}^{1 / 2}(a, b)}^{2} & =\int_{\mathbb{R}} \int_{\mathbb{R}} \frac{|\tilde{v}(x)-\tilde{v}(y)|^{2}}{|x-y|^{2}} d x d y \\
& =\int_{a}^{b} \int_{a}^{b} \frac{|v(x)-v(y)|^{2}}{|x-y|^{2}} d x d y+2 \int_{a}^{b}|v(x)|^{2}\left(\frac{1}{x-a}+\frac{1}{b-x}\right) d x \\
& =|v|_{H^{1 / 2}(a, b)}^{2}+2 \int_{a}^{b}|v(x)|^{2}\left(\frac{1}{x-a}+\frac{1}{b-x}\right) d x
\end{aligned}
$$

Note that the norm defined by (3.2) is invariant with respect to translation and scaling.

It is well known (cf. [18, 23]) that

$$
H_{00}^{1 / 2}(a, b)=\left[L_{2}(a, b), H_{0}^{1}(a, b)\right]_{1 / 2}
$$

with an equivalent norm, where $\left[L_{2}(a, b), H_{0}^{1}(a, b)\right]_{1 / 2}$ is the interpolation space halfway between $L_{2}(a, b)$ and $H_{0}^{1}(a, b)$ obtained by the complex method (cf. [1], [23], [14]).

Let $\phi$ be a continuous function defined on $(a, b)$ which is piecewise linear with respect to the uniform subdivision of mesh size $\rho$, and $\phi(a)=\phi(b)=0$. The following estimate (cf. [3], [7]) is crucial to the condition number estimates for nonoverlapping domain decomposition methods in two dimensions:

$$
\|\phi\|_{L_{\infty}(a, b)} \leq C(1+|\ln \rho|)^{1 / 2}|\phi|_{H_{00}^{1 / 2}(a, b)} .
$$

Therefore the first step towards proving the sharpness of (2.14) and (2.20) is to produce a piecewise linear function for which the estimate (3.4) is sharp. This will be achieved through the interpolation of finite sine series by piecewise linear functions.

In order to avoid the proliferation of constants, we will henceforth use the notation $A \lesssim B$ (or $B \gtrsim A$ ) to represent the statement that $A \leq$ constant $\times B$, where the constant is a universal constant (i.e., independent of any parameters). The notation $A \approx B$ means that $A \lesssim B$ and $A \gtrsim B$.

Let $v=\sum_{n=1}^{\infty} v_{n} \sin ((n \pi / \ell) x)$ be an arbitrary function in $L_{2}(0, \ell)$. By Parseval's identity, we have

$$
\|v\|_{L_{2}(0, \ell)}^{2} \approx \sum_{n=1}^{\infty}\left|v_{n}\right|^{2}
$$

where the scaling invariant norm $\|\cdot\|_{L_{2}(0, \ell)}$ is defined in (1.5). Similarly, $v$ belongs to $H_{0}^{1}(0, \ell)$ if and only if $\sum_{n=1}^{\infty} n^{2}\left|v_{n}\right|^{2}<\infty$, and we have

$$
\|v\|_{H^{1}(0, \ell)}^{2} \approx \sum_{n=1}^{\infty} n^{2}\left|v_{n}\right|^{2}
$$

where the scaling invariant norm $\|\cdot\|_{H^{1}(0, \ell)}$ is defined in (1.7).

Let the space $\mathcal{F}_{s}$ be defined by

$$
\mathcal{F}_{s}=\left\{v \in L_{2}(0, \ell): v=\sum_{n=1}^{\infty} v_{n} \sin ((n \pi / \ell) x) \text { and } \sum_{n=1}^{\infty} n^{2 s}\left|v_{n}\right|^{2}<\infty\right\},
$$


with the norm $\|\cdot\|_{s}$ defined by

$$
\|v\|_{s}^{2}=\sum_{n=1}^{\infty} n^{2 s}\left|v_{n}\right|^{2} .
$$

Then the spaces $\mathcal{F}_{s}$ form a Hilbert scale (cf. [14]) and we have

$$
\mathcal{F}_{1 / 2}=\left[\mathcal{F}_{0}, \mathcal{F}_{1}\right]_{1 / 2} \text {. }
$$

Since $\mathcal{F}_{0}=L_{2}(0, \ell)$ and $\mathcal{F}_{1}=H_{0}^{1}(0, \ell)$, the following lemma is an immediate consequence of (3.3), (3.5)-(3.9) and interpolation.

Lemma 3.1. A function $v=\sum_{n=1}^{\infty} v_{n} \sin ((n \pi / \ell) x) \in L_{2}(0, \ell)$ belongs to $H_{00}^{1 / 2}(0, \ell)$ if and only if $\sum_{n=1}^{\infty} n\left|v_{n}\right|^{2}<\infty$, and we have $|v|_{H_{00}^{1 / 2}(0, \ell)}^{2} \approx \sum_{n=1}^{\infty} n\left|v_{n}\right|^{2}$.

Let $N=2^{k}(k=0,1,2,3, \ldots)$ and the function $G_{N}$ on $(0,1)$ be defined by

$$
G_{N}(x)=\sum_{n=1}^{N}\left(\frac{1}{4 n-3}\right) \sin ((4 n-3) \pi x) .
$$

The properties of $G_{N}$ are summarized in the following lemma.

Lemma 3.2. The function $G_{N}$ is symmetric with respect to the midpoint of $(0,1)$, where it attains its maximum in absolute value. Moreover, we have

$$
\begin{aligned}
\left\|G_{N}\right\|_{H^{1}(0,1)}^{2} & \approx N, \\
\left|G_{N}\right|_{H_{00}^{1 / 2}(0,1)}^{2} & \approx 1+\ln N, \\
\left\|G_{N}\right\|_{L_{\infty}(0,1)} & =G_{N}(1 / 2) \approx 1+\ln N .
\end{aligned}
$$

Proof. The symmetry of $G_{N}$ is straightforward, and (3.13) follows from Lemma 6.1 in Section 6. The estimate (3.11) follows from (3.6), and (3.12) follows from Lemma 3.1 and Lemma 6.1.

Let $\mathcal{L}_{\rho}(0,1)$ be the space of continuous functions which are piecewise linear with respect to the uniform subdivision of $(0,1)$ of mesh size $\rho$, and $\hat{\Pi}_{\rho}: H^{1}(0,1) \longrightarrow$ $\mathcal{L}_{\rho}(0,1)$ be the nodal interpolation operator.

Lemma 3.3. Let $\rho=1 / N$ and $\hat{g}_{\rho}=\hat{\Pi}_{\rho} G_{N}$. Then $\hat{g}_{\rho}$ is symmetric with respect to the midpoint of $(0,1)$ and we have

$$
\begin{aligned}
& \left\|\hat{g}_{\rho}\right\|_{L_{\infty}(0,1)}=g_{h}(1 / 2) \approx 1+|\ln \rho|, \\
& \left|\hat{g}_{\rho}\right|_{H_{00}^{1 / 2}(0,1)}^{2} \approx 1+|\ln \rho| .
\end{aligned}
$$

Proof. The symmetry of $\hat{g}_{\rho}$ and (3.14) follows immediately from the symmetry of $G_{N}$ and (3.13).

We have the following interpolation error estimate (cf. [6, 4]) for the interpolation operator $\hat{\Pi}_{\rho}$ :

$$
\left\|\zeta-\hat{\Pi}_{\rho} \zeta\right\|_{L_{2}(0,1)}+\rho\left|\zeta-\hat{\Pi}_{\rho} \zeta\right|_{H^{1}(0,1)} \lesssim \rho|\zeta|_{H^{1}(0,1)} \quad \forall \zeta \in H^{1}(0,1) .
$$

In view of (3.3), we can interpolate (3.16) to obtain

$$
\left|\zeta-\hat{\Pi}_{\rho} \zeta\right|_{H_{00}^{1 / 2}(0,1)} \lesssim \rho^{1 / 2}|\zeta|_{H^{1}(0,1)} \quad \forall \zeta \in H^{1}(0,1) .
$$


By (3.17) and (3.11) we have

$$
\left|G_{N}-\hat{g}_{\rho}\right|_{H_{\mathrm{OO}}^{1 / 2}(0,1)} \lesssim 1
$$

The estimate (3.15) then follows from (3.12) and (3.18).

Combining (3.14) and (3.15), we have

$$
\left\|\hat{g}_{\rho}\right\|_{L_{\infty}(0,1)} \approx(1+|\ln \rho|)^{1 / 2}\left|\hat{g}_{\rho}\right|_{H_{00}^{1 / 2}(0,1)} .
$$

In other words, we have constructed a continuous piecewise linear function for which (3.4) is sharp.

Remark 3.4. A related estimate is (cf. [3], 7])

$$
\|\phi\|_{L_{\infty}(a, b)} \lesssim(1+|\ln \rho|)^{1 / 2}\|\phi\|_{H^{1 / 2}(a, b)},
$$

which holds for any continuous function $\phi$ on $(a, b)$ that is piecewise linear with respect to the uniform subdivision of mesh size $\rho$. The estimate (3.19) shows that (3.20) is also sharp, since

$$
\left\|\hat{g}_{\rho}\right\|_{L_{\infty}(0,1)} \approx(1+|\ln \rho|)^{1 / 2}\left\|\hat{g}_{\rho}\right\|_{H_{00}^{1 / 2}(0,1)} \geq(1+|\ln \rho|)^{1 / 2}\left\|\hat{g}_{\rho}\right\|_{H^{1 / 2}(0,1)} .
$$

The sharpness of (3.20) was also investigated numerically in [13].

Remark 3.5. Let $v$ be a discrete harmonic function defined on the unit square $D$ with vertices $(0,0),(1,0),(1,1)$ and $(0,1)$ with respect to the uniform triangulation of mesh size $\rho$ such that $v(x, 0)=\hat{g}_{\rho}(x)$ for $0 \leq x \leq 1$ and $v$ vanishes on the other three sides. Then it follows from Lemma 2.5 and Lemma 3.3 that

$$
\|v\|_{L_{\infty}(D)} \gtrsim(1+|\ln \rho|)^{1 / 2}\|v\|_{H^{1}(D)},
$$

which implies that the discrete Sobolev inequality (cf. [3]) is sharp.

The following corollary is obtained from Lemma 3.3 by scaling.

Corollary 3.6. Let $g_{h}$ be the function on $[-H, H]$ defined by

$$
g_{h}(x)=\hat{g}_{\rho}\left(\frac{x+H}{2 H}\right) \quad \text { for } \quad \rho=\left(\frac{h}{2 H}\right) .
$$

Then $g_{h}$ has the following properties:

(i) $g_{h}$ is piecewise linear with respect to the uniform subdivision of $[-H, H]$ of mesh size $h$, and $g_{h}(-H)=g_{h}(H)=0$;

(ii) $g_{h}$ is symmetric with respect to the midpoint 0 ;

(iii) $\left\|g_{h}\right\|_{L_{\infty}(-H, H)}$ and $\left|g_{h}\right|_{H_{00}^{1 / 2}(-H, H)}$ are estimated by

$$
\begin{aligned}
& g_{h}(0)=\left\|g_{h}\right\|_{L_{\infty}(-H, H)} \approx 1+\ln \frac{H}{h}, \\
& \left|g_{h}\right|_{H_{00}^{1 / 2}(-H, H)}^{2} \approx 1+\ln \frac{H}{h} .
\end{aligned}
$$

The piecewise linear function $g_{h}$ will play a key role in the constructions in Sections 4 and 5 , and we will also need the estimates for some related piecewise linear functions. First we state two technical lemmas (Lemma 3.7 and Lemma 3.8) on the unit interval whose proofs are deferred to Section 6.

Recall that $\hat{\Pi}_{\frac{1}{2}}: H^{1}(0,1) \longrightarrow \mathcal{L}_{\frac{1}{2}}(0,1)$ is the nodal interpolation operator with respect to the subdivision $\{0,1 / 2,1\}$. 
Lemma 3.7. The following estimate holds:

$$
\left|\hat{g}_{\rho}-\hat{\Pi}_{\frac{1}{2}} \hat{g}_{\rho}\right|_{H_{00}^{1 / 2}(0,1 / 2)}^{2}=\left|\hat{g}_{\rho}-\hat{\Pi}_{\frac{1}{2}} \hat{g}_{\rho}\right|_{H_{00}^{1 / 2}(1 / 2,1)}^{2} \approx(1+|\ln \rho|)^{3} .
$$

Let $\hat{p}_{\rho}$, a continuous function on $\left[0, \frac{1}{2}+\rho\right]$ which is piecewise linear with respect to the uniform subdivision of mesh size $\rho$, be defined by

$$
\left.\hat{p}_{\rho}\right|_{\left[0, \frac{1}{2}-\rho\right]}=\left.\hat{g}_{\rho}\right|_{\left[0, \frac{1}{2}-\rho\right]}, \quad \hat{p}_{\rho}\left(\frac{1}{2}\right)=\frac{1}{2} \hat{g}_{\rho}\left(\frac{1}{2}\right) \quad \text { and } \quad \hat{p}_{\rho}\left(\frac{1}{2}+\rho\right)=0
$$

and similarly $\hat{q}_{\rho}$, a continuous piecewise linear function on $\left[\frac{1}{2}-\rho, 1\right]$, be defined by

$$
\left.\hat{q}_{\rho}\right|_{\left[\frac{1}{2}+\rho, 1\right]}=\left.\hat{g}_{\rho}\right|_{\left[\frac{1}{2}+\rho, 1\right]}, \quad \hat{q}_{\rho}\left(\frac{1}{2}\right)=\frac{1}{2} \hat{g}_{\rho}\left(\frac{1}{2}\right) \quad \text { and } \quad \hat{q}_{\rho}\left(\frac{1}{2}-\rho\right)=0 .
$$

Lemma 3.8. The following estimate holds:

$$
\left|\hat{p}_{\rho}\right|_{H_{00}^{1 / 2}\left(0, \frac{1}{2}+\rho\right)}^{2}=\left|\hat{q}_{\rho}\right|_{H_{00}^{1 / 2}\left(\frac{1}{2}-\rho, 1\right)}^{2} \approx(1+|\ln \rho|)^{3} .
$$

Let $\Pi_{H}$ be the nodal piecewise linear interpolation operator with respect to the subdivision $\{-H, 0, H\}$ of $[-H, H]$. The following corollary is obtained from Lemma 3.7 by scaling.

Corollary 3.9. Let $g_{h}$ be the function defined by (3.21). Then we have

$$
\left|g_{h}-\Pi_{H} g_{h}\right|_{H_{00}^{1 / 2}(-H, 0)}^{2}=\left|g_{h}-\Pi_{H} g_{h}\right|_{H_{00}^{1 / 2}(0, H)}^{2} \approx\left(1+\ln \frac{H}{h}\right)^{3} .
$$

Let $p_{h}$, a continuous function on $(-H, h)$ which is piecewise linear with respect to the uniform subdivision of mesh size $h$, be defined by

$$
\left.p_{h}\right|_{[-H,-h]}=\left.g_{h}\right|_{[-H,-h]}, \quad p_{h}(0)=\frac{1}{2} g_{h}(0) \quad \text { and } \quad p_{h}(h)=0,
$$

and similarly $q_{h}$, a continuous piecewise linear function on $[-h, H]$, be defined by

$$
\left.q_{h}\right|_{[h, H]}=\left.g_{h}\right|_{[h, H]}, \quad q_{h}(0)=\frac{1}{2} g_{h}(0) \quad \text { and } \quad q_{h}(-h)=0 .
$$

The functions $p_{h}$ and $q_{h}$ are scaled analogs of $\hat{p}_{\rho}$ and $\hat{q}_{\rho}$, and the following corollary is a scaled version of Lemma 3.8 .

Corollary 3.10. The following estimate holds for the functions $p_{h}$ and $q_{h}$ defined by (3.28) and (3.29):

$$
\left|p_{h}\right|_{H_{00}^{1 / 2}(-H, h)}^{2}=\left|q_{h}\right|_{H_{00}^{1 / 2}(-h, H)}^{2} \approx\left(1+\ln \frac{H}{h}\right)^{3} .
$$

\section{LOWER BOUND FOR THE BPS PRECONDITIONER}

According to (1.3), (1.4), (2.7), (2.9), (2.11) and (2.13), we have the following characterizations of $\lambda_{\max }\left(B_{B P S} S_{h}\right)$ and $\lambda_{\min }\left(B_{B P S} S_{h}\right)$ :

$$
\begin{aligned}
\lambda_{\min }\left(B_{B P S} S_{h}\right) & =\min _{0 \neq v \in V_{h}(\Gamma)} \frac{a(v, v)}{a\left(v_{H}, v_{H}\right)+\sum_{\ell=1}^{L} a\left(v_{\ell}, v_{\ell}\right)}, \\
\lambda_{\max }\left(B_{B P S} S_{h}\right) & =\max _{0 \neq v \in V_{h}(\Gamma)} \frac{a(v, v)}{a\left(v_{H}, v_{H}\right)+\sum_{\ell=1}^{L} a\left(v_{\ell}, v_{\ell}\right)},
\end{aligned}
$$

where $v_{H} \in V_{H}$ and $v_{\ell} \in V_{h}\left(E_{\ell}\right)(1 \leq \ell \leq L)$ form the unique decomposition $v=I_{H} v_{H}+\sum_{\ell=1}^{L} v_{\ell}$. 


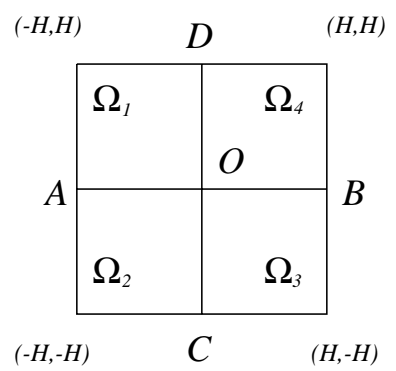

Figure 4.

Lemma 4.1. We have $\lambda_{\max }\left(B_{B P S} S_{h}\right) \geq 1$.

Proof. Let $0 \neq v_{*}$ belong to one of the edge spaces, say, $v_{*} \in V_{h}\left(E_{1}\right)$. The decomposition of $v_{*}$ is given by $v_{H}=0, v_{1}=v_{*}$ and $0=v_{2}=\cdots=v_{L}=0$. Hence we have

$$
\frac{a\left(v_{*}, v_{*}\right)}{a\left(v_{H}, v_{H}\right)+\sum_{\ell=1}^{L} a\left(v_{\ell}, v_{\ell}\right)}=\frac{a\left(v_{*}, v_{*}\right)}{a\left(v_{*}, v_{*}\right)}=1,
$$

and the lemma follows from (4.2).

Lemma 4.2. We have $\lambda_{\min }\left(B_{B P S} S_{h}\right) \lesssim[1+\ln (H / h)]^{-2}$.

Proof. We need to construct $0 \neq v_{\dagger} \in V_{h}(\Gamma)$ such that

$$
a\left(v_{\dagger}, v_{\dagger}\right) \lesssim\left[1+\ln \frac{H}{h}\right]^{-2}\left[a\left(v_{H}, v_{H}\right)+\sum_{\ell=1}^{L} a\left(v_{\ell}, v_{\ell}\right)\right]
$$

holds for the unique decomposition $v=I_{H} v_{H}+\sum_{\ell=1}^{L} v_{\ell}$, where $v_{H} \in V_{H}$ and $v_{\ell} \in V_{h}\left(E_{\ell}\right)$ for $1 \leq \ell \leq L$. The lemma then follows from (4.1) and (4.3).

The definition of $v_{\dagger}$ involves the four subdomains $\Omega_{1}, \Omega_{2}, \Omega_{3}$ and $\Omega_{4}$ neighboring the center of $\Omega$, whose vertices are given by $(0,0),(0, \pm H),( \pm H, 0)$ and $( \pm H, \pm H)$ (cf. Figure 4).

Let $g_{h}$ be the function defined by (3.21). The function $v_{\dagger} \in V_{h}(\Gamma)$ is defined to be 0 on $\Gamma$ except on the line segments $A B$ and $C D$, where it is given by

$$
\begin{array}{ll}
v_{\dagger}(x, 0)=g_{h}(x) & \text { for }-H \leq x \leq H, \\
v_{\dagger}(0, y)=g_{h}(y) & \text { for }-H \leq y \leq H .
\end{array}
$$

It is clear that $v_{\dagger}$ vanishes outside $\Omega_{1} \cup \Omega_{2} \cup \Omega_{3} \cup \Omega_{4}$. In the unique decomposition of $v_{\dagger}$, the coarse grid function $v_{H}$ is just the nodal interpolant of $v_{\dagger}$ in the coarse grid space $V_{H}$, and the only nontrivial edge space functions are associated with the four edges $E_{1}=O A, E_{2}=O B, E_{3}=O C$, and $E_{4}=O D$.

By Lemma 2.5, we have

$$
a\left(v_{\dagger}, v_{\dagger}\right)=\sum_{j=1}^{4}\left|v_{\dagger}\right|_{H^{1}\left(\Omega_{j}\right)}^{2} \approx \sum_{j=1}^{4}\left|v_{\dagger}\right|_{H^{1 / 2}\left(\partial \Omega_{j}\right)}^{2},
$$

and from (1.11), (3.2), (4.4), (4.5), and the symmetry of $g_{h}$ we find

$$
\left|v_{\dagger}\right|_{H^{1 / 2}\left(\partial \Omega_{j}\right)}^{2} \approx\left|g_{h}\right|_{H_{00}^{1 / 2}(-H, H)}^{2} \quad \text { for } 1 \leq j \leq 4 .
$$


Combining (3.23), (4.6) and (4.7), we conclude that

$$
a\left(v_{\dagger}, v_{\dagger}\right) \approx 1+\ln \frac{H}{h} .
$$

Since $v_{H} \in V_{H}$ vanishes at all the vertices of $\mathcal{T}_{H}$ except the origin, where $v_{H}(0,0)=g_{h}(0)$, a simple calculation shows that $a\left(v_{H}, v_{H}\right) \approx|v(0)|^{2}=\left|g_{h}(0)\right|^{2}$, and then it follows from (3.22) that

$$
a\left(v_{H}, v_{H}\right) \approx\left(1+\ln \frac{H}{h}\right)^{2} .
$$

Finally we estimate $a\left(v_{\ell}, v_{\ell}\right)$ for the edge functions $v_{\ell}(1 \leq \ell \leq 4)$. Since $v_{\dagger}=$ $I_{H} v_{H}+\sum_{\ell=1}^{4} v_{\ell}$, on the edge $E_{\ell}$ the function $v_{\ell}$ equals the difference between $v_{\dagger}$ and its coarse grid interpolant, and $v_{\ell}$ vanishes on $\Gamma \backslash E_{\ell}$.

Let $\Omega_{\ell_{1}}$ and $\Omega_{\ell_{2}}$ be the two subdomains neighboring $E_{\ell}$. We have, by Lemma 2.5, (1.11) and (3.2),

$$
a\left(v_{\ell}, v_{\ell}\right)=\left|v_{\ell}\right|_{H^{1}\left(\Omega_{\ell_{1}}\right)}^{2}+\left|v_{\ell}\right|_{H^{1}\left(\Omega_{\ell_{2}}\right)}^{2} \approx\left|v_{\ell}\right|_{H_{00}^{1 / 2}\left(E_{\ell}\right)}^{2},
$$

and then Corollary 3.9 and (4.4)-(4.5) imply that

$$
a\left(v_{\ell}, v_{\ell}\right) \approx\left(1+\ln \frac{H}{h}\right)^{3} \quad \text { for } 1 \leq \ell \leq 4 .
$$

The estimate (4.3) (and hence the lemma) follows from (4.8)-(4.10).

Combining Lemmas 4.1 and 4.2, we have the following theorem on the lower bound of $\kappa\left(B_{B P S} S_{h}\right)$.

Theorem 4.3. For the model problem described in Section 2, we have

$$
\kappa\left(B_{B P S} S_{h}\right) \geq c\left(1+\ln \frac{H}{h}\right)^{2},
$$

where $c$ is independent of $h, H$ and $J$.

Remark 4.4. The proof of Lemma 4.2 (and hence Theorem 4.3) requires at least one cross point, which is satisfied by the model problem in Section 2. It also agrees with the fact that $\kappa\left(B_{B P S} S_{h}\right) \lesssim 1$ when there are no cross points (cf. [3]).

\section{LOWER BOUnd FOR THE NEUMANn-Neumann PRECONDitioner}

According to (1.3), (1.4), (2.7), (2.9), and (2.18), we have the following characterizations of $\lambda_{\max }\left(B_{N N} S_{h}\right)$ and $\lambda_{\min }\left(B_{N N} S_{h}\right)$ :

$$
\begin{aligned}
& \lambda_{\min }\left(B_{N N} S_{h}\right)=\min _{0 \neq v \in V_{h}(\Gamma)} \frac{a(v, v)}{\min _{\substack{v=I_{H} v_{H}+\sum_{j=1}^{J} \hat{I}_{j} v_{j} \\
v_{H} \in V_{H}, v_{j} \in V_{h}\left(\Gamma_{j}\right)}}\left[a\left(v_{H}, v_{H}\right)+\sum_{j=1}^{J} \hat{a}_{j}\left(v_{j}, v_{j}\right)\right]}, \\
& \lambda_{\max }\left(B_{N N} S_{h}\right)=\max _{0 \neq v \in V_{h}(\Gamma)} \frac{a(v, v)}{\min _{\substack{v=I_{H} v_{H}+\sum_{j=1}^{J} \hat{I}_{j} v_{j} \\
v_{H} \in V_{H}, v_{j} \in V_{h}\left(\Gamma_{j}\right)}}\left[a\left(v_{H}, v_{H}\right)+\sum_{j=1}^{J} \hat{a}_{j}\left(v_{\ell}, v_{\ell}\right)\right]}
\end{aligned}
$$

This time we will first establish an upper bound for $\lambda_{\min }\left(B_{N N} S_{h}\right)$. We begin with the construction of a piecewise linear function on $(0, H)$. 


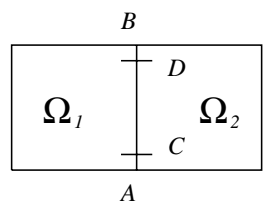

FIGURE 5 .

Lemma 5.1. There exists a continuous function $\psi$ on $(0, H)$, not identically zero, which is piecewise linear with respect to the uniform subdivision $\mathbb{S}$ of mesh size $H / 8$, and has the following properties:

(i) $\psi(x)=0$ for $x \notin\left(\frac{H}{4}, \frac{3 H}{4}\right)$,

(ii) $(\psi, w)_{H^{1 / 2}(H / 8,7 H / 8)}=0$ for any linear polynomial $w$ on $(H / 8,7 H / 8)$.

Proof. Let $\mathcal{V}$ be the space of continuous piecewise linear functions associated with $\mathbb{S}$ that satisfy (i). Then $\operatorname{dim} \mathcal{V}$ equals three, while there are only two linearly independent conditions in (ii).

Lemma 5.2. We have $\lambda_{\min }\left(B_{N N} S_{h}\right) \lesssim 1$.

Proof. For $H / h \leq 4$, this is a consequence of the estimate (cf. [9, 10, [1]) $\lambda_{\max }\left(B_{N N} S_{h}\right) \lesssim\left(1+\ln \frac{H}{h}\right)^{2}$.

For $H / h \geq 8$, we construct a function $v_{\dagger} \in V_{h}(\Gamma)$ as follows. Let $A B$ be the line segment connecting $(0,0)$ to $(0, H)$, and denote by $\Omega_{1}$ and $\Omega_{2}$ the two neighboring subdomains (cf. Figure 5). We define $v_{\dagger}$ to be 0 on $\Gamma$ except on the line segment $A B$, and on $A B$ we have

$$
v_{\dagger}(0, y)=\psi(y) \quad \text { for } 0 \leq y \leq H,
$$

where $\psi$ is the function from Lemma 5.1. Clearly $v_{\dagger}$ vanishes outside $\Omega_{1} \cup \Omega_{2}$, and we have, by Lemma 2.5 and Lemma 5.1 (i),

$$
a\left(v_{\dagger}, v_{\dagger}\right)=\left|v_{\dagger}\right|_{H^{1}\left(\Omega_{1}\right)}^{2}+\left|v_{\dagger}\right|_{H^{1}\left(\Omega_{2}\right)}^{2} \approx\left\|v_{\dagger}\right\|_{H^{1 / 2}(C D)}^{2},
$$

where $C=(0, H / 8)$ and $D=(0,7 H / 8)$ (cf. Figure 5$)$.

Consider now an arbitrary decomposition $v_{\dagger}=I_{H} v_{H}+\sum_{j=1}^{J} \hat{I}_{j} v_{j}$, where $v_{H} \in$ $V_{H}$ and $v_{j} \in V_{h}\left(\Gamma_{j}\right)$. On the line segment $C D$, by (2.8) and (2.17), $I_{H} v_{H}=v_{H}$ is a linear polynomial, and

$$
\left.\left(v_{\dagger}-v_{H}\right)\right|_{C D}=\left.\left(\frac{v_{1}+v_{2}}{2}\right)\right|_{C D} .
$$

It follows from Lemma 5.1 (ii), (5.3) and (5.5) that

$$
\left\|v_{\dagger}\right\|_{H^{1 / 2}(C D)}^{2} \leq\left\|v_{\dagger}-v_{H}\right\|_{H^{1 / 2}(C D)}^{2} \lesssim\left\|v_{1}\right\|_{H^{1 / 2}(C D)}^{2}+\left\|v_{2}\right\|_{H^{1 / 2}(C D)}^{2} .
$$

Since $\hat{a}_{j}(\cdot, \cdot)=\|\cdot\|_{H^{1}\left(\Omega_{j}\right)}^{2}$ (cf. (1.7) and (2.15)), it follows from (5.6) and the trace theorem (cf. [23]) that

$$
\left\|v_{\dagger}\right\|_{H^{1 / 2}(C D)}^{2} \lesssim \hat{a}_{1}\left(v_{1}, v_{1}\right)+\hat{a}_{2}\left(v_{2}, v_{2}\right) .
$$

Combining (5.4) and (5.7), we find $a\left(v_{\dagger}, v_{\dagger}\right) \lesssim a\left(v_{H}, v_{H}\right)+\sum_{j=1}^{J} \hat{a}_{j}\left(v_{j}, v_{j}\right)$. Since this last estimate holds for any decomposition of $v_{\dagger}$, the lemma follows from (5.1).

Now we prove a lower bound for $\lambda_{\max }\left(B_{N N} S_{h}\right)$. 
Lemma 5.3. We have $\lambda_{\max }\left(B_{N N} S_{h}\right) \gtrsim[1+\ln (H / h)]^{2}$.

Proof. We need to construct $0 \neq v_{*} \in V_{h}(\Gamma)$ such that, for one decomposition $v_{*}=I_{H} v_{H}+\sum_{j=1}^{J} \hat{I}_{j} v_{j}$, where $v_{H} \in V_{H}$ and $v_{j} \in V_{h}\left(\Gamma_{j}\right)$, we have

$$
a\left(v_{*}, v_{*}\right) \gtrsim\left(1+\ln \frac{H}{h}\right)^{2}\left[a\left(v_{H}, v_{H}\right)+\sum_{j=1}^{J} \hat{a}_{j}\left(v_{j}, v_{j}\right)\right] .
$$

The lemma then follows from (5.2) and (5.8).

The construction of $v_{*}$ again involves the four subdomains neighboring the center of $\Omega$ (cf. Figure 4). We define $w \in V_{h}\left(\Gamma_{1}\right)$ to be 0 on $\Gamma_{1}$ except on the two line segments $O A$ and $O D$, where we define

$$
\begin{aligned}
& w(x, 0)=g_{h}(x) \quad \text { for } \quad-H \leq x \leq 0, \\
& w(0, y)=g_{h}(y) \quad \text { for } \quad 0 \leq y \leq H \text {. }
\end{aligned}
$$

The function $v_{*}$ is then defined to be $\hat{I}_{1} w$, and we choose the decomposition

$$
0=v_{H}=v_{2}=v_{3}=\cdots=v_{J} \quad \text { and } \quad v_{1}=w .
$$

By Lemma 2.5 and Corollary 3.6, we have

$$
\hat{a}_{1}\left(v_{1}, v_{1}\right) \approx|w|_{H^{1 / 2}\left(\partial \Omega_{1}\right)}^{2} \approx\left|g_{h}\right|_{H_{00}^{1 / 2}(-H, H)}^{2} \approx 1+\ln \frac{H}{h} .
$$

From the definition of $\hat{I}_{1}$ (cf. (2.17)) and (5.9), it is clear that $v_{*}$ vanishes outside $\Omega_{1} \cup \Omega_{2} \cup \Omega_{3} \cup \Omega_{4}$. It follows that

$$
a\left(v_{*}, v_{*}\right)=\sum_{j=1}^{4}\left|v_{*}\right|_{H^{1}\left(\Omega_{j}\right)}^{2} .
$$

Using (2.17), (3.28), Corollary 3.10, (5.9), and Lemma 2.5, we have

$$
\left|v_{*}\right|_{H^{1}\left(\Omega_{2}\right)}^{2} \approx\left|v_{*}\right|_{H^{1 / 2}\left(\partial \Omega_{2}\right)}^{2} \approx\left|p_{h}\right|_{H_{00}^{1 / 2}(-H, h)}^{2} \approx\left(1+\ln \frac{H}{h}\right)^{3} .
$$

It follows from $(5.11)-(5.13)$ that $a\left(v_{*}, v_{*}\right) \gtrsim\left(1+\ln \frac{H}{h}\right)^{2} \hat{a}_{1}\left(v_{1}, v_{1}\right)$, which implies (5.8) for the decomposition in (5.10) and hence the lemma.

Combining Lemmas 5.2 and 5.3, we have the following theorem on the lower bound of $\kappa\left(B_{N N} S_{h}\right)$.

Theorem 5.4. For the model problem described in Section 2, we have

$$
\kappa\left(B_{N N} S_{h}\right) \geq c\left(1+\ln \frac{H}{h}\right)^{2},
$$

where $c$ is independent of $h, H$ and $J$. 


\section{Proofs of the tWo TECHNICAL LEMMAS}

We will present the proofs of Lemma 3.7 and Lemma 3.8 in this section. We begin with an elementary lemma from calculus.

Lemma 6.1. Let $\psi$ be a positive, continuous and decreasing function defined on $(0, \infty)$. Then we have

$$
\sum_{k=1}^{K} f(k) \approx f(1)+\int_{1}^{K} f(x) d x
$$

Proof. Using Riemann sums and the sign and monotonicity of $f(x)$, we have

$$
\sum_{k=1}^{K} f(k) \leq f(1)+\int_{1}^{K} f(x) d x \quad \text { and } \quad \int_{1}^{K} f(x) d x \leq \sum_{k=1}^{K-1} f(k) .
$$

The coefficients of the sine series $\sum_{n=1}^{\infty} \alpha_{n} \sin (2 n \pi x)$ of the function $G_{N}-\hat{\Pi}_{\frac{1}{2}} G_{N}$ on the interval $(0,1 / 2)$ are given by

$$
\begin{aligned}
\alpha_{n} & =4 \int_{0}^{1 / 2}\left[G_{N}-\hat{\Pi}_{\frac{1}{2}} G_{N}\right] \sin (2 n \pi x) d x \\
& =\frac{2}{n \pi} \int_{0}^{1 / 2}\left[G_{N}-\hat{\Pi}_{\frac{1}{2}} G_{N}\right]^{\prime} \cos (2 n \pi x) d x .
\end{aligned}
$$

Note that $\left(\hat{\Pi}_{\frac{1}{2}} G_{N}\right)(x)=\left[2 G_{N}(1 / 2)\right] x$ on $(0,1 / 2)$. Hence we have, by $(3.10)$,

$$
\left[G_{N}-\hat{\Pi}_{\frac{1}{2}} G_{N}\right]^{\prime}(x)=\pi\left[\sum_{m=1}^{N} \cos ((4 m-3) \pi x)\right]-2 G_{N}(1 / 2)
$$

for $0<x<\frac{1}{2}$.

We can therefore write

$$
\alpha_{n}=\frac{(-1)^{n}}{\pi} \frac{1}{n} \sum_{m=1}^{N} a_{m, n}
$$

where

$$
a_{m, n}=\frac{1}{2 n+4 m-3}-\frac{1}{2 n-4 m+3} .
$$

Lemma 6.2. It holds that $\sum_{n=3 N+1}^{\infty} \frac{1}{n}\left(\sum_{m=1}^{N} a_{m, n}\right)^{2} \lesssim 1$.

Proof. From (6.3) we have, for $n>3 N,\left|\sum_{m=1}^{N} a_{m, n}\right| \lesssim\left|\sum_{m=1}^{N} \frac{1}{n}\right| \lesssim \frac{N}{n}$. Then Lemma 6.1 implies that

$$
\sum_{n=3 N+1}^{\infty} \frac{1}{n}\left(\sum_{m=1}^{N} a_{m, n}\right)^{2} \lesssim N^{2} \sum_{n=3 N+1}^{\infty} \frac{1}{n^{3}} \approx 1 .
$$

Lemma 6.3. It holds that $\sum_{n=N}^{3 N} \frac{1}{n}\left(\sum_{m=1}^{N} a_{m, n}\right)^{2} \lesssim(1+\ln N)^{2}$. 
Proof. For $N \leq n \leq 3 N$, we have, by Lemma 6.1,

$$
\sum_{m=1}^{N} \frac{1}{2 n+4 m-3} \approx \frac{1}{2 n+1}+\frac{1}{4} \ln \left(\frac{2 n+4 N-3}{2 n+1}\right) \approx 1
$$

and

$$
\sum_{m=1}^{N}\left|\frac{1}{2 n-4 m+3}\right| \lesssim \sum_{j=1}^{6 N-1} \frac{1}{j} \approx 1+\ln N .
$$

It follows from these two estimates, (6.3) and Lemma 6.1 that

$$
\sum_{n=N}^{3 N} \frac{1}{n}\left(\sum_{m=1}^{N} a_{m, n}\right)^{2} \lesssim(1+\ln N)^{2} \sum_{n=N}^{3 N} \frac{1}{n} \approx(1+\ln N)^{2} .
$$

Lemma 6.4. It holds that $\sum_{n=1}^{N-1} \frac{1}{n}\left(\sum_{m=1}^{N} a_{m, n}\right)^{2} \approx(1+\ln N)^{3}$.

Proof. For $1 \leq n \leq N-1$, we can write, by (6.3),

$$
\sum_{m=1}^{N} a_{m, n}=c_{n}-b_{n}
$$

where

$$
b_{n}=\sum_{m=1}^{n} \frac{1}{2 n-4 m+3}
$$

and

$$
c_{n}=\left[\sum_{m=1}^{N} \frac{1}{2 n+4 m-3}\right]-\left[\sum_{m=n+1}^{N} \frac{1}{2 n-4 m+3}\right] .
$$

We claim that

$$
\left|b_{n}\right| \lesssim 1 \quad \text { for } 1 \leq n \leq N
$$

Assume first that $n$ is even. Then we have, for $n=2 \ell$,

$$
\begin{aligned}
\left|b_{n}\right| & =\left|\sum_{m=1}^{\ell}\left(\frac{1}{2 n-4 m+3}+\frac{1}{2 n-4(m+\ell)+3}\right)\right| \\
& =\left|\sum_{m=1}^{\ell}\left(\frac{1}{2 n-4(\ell-m+1)+3}+\frac{1}{2 n-4(m+\ell)+3}\right)\right| \\
& =\left|\sum_{m=1}^{\ell}\left(\frac{1}{4 m-1}-\frac{1}{4 m-3}\right)\right| \lesssim 1 .
\end{aligned}
$$

On the other hand, when $n=2 \ell-1$, we also find by using the previous case that

$$
\left|b_{n}\right|=\left|\left(\sum_{m=1}^{n+1} \frac{1}{2 n-4 m+3}\right)+\frac{1}{2 n+1}\right| \lesssim 1 .
$$


Next we estimate $\left|c_{n}\right|$. Observe that $-\sum_{m=n+1}^{N} \frac{1}{2 n-4 m+3}=\sum_{m=1}^{N-n} \frac{1}{2 n+4 m-3}$, and hence

$$
\sum_{m=1}^{N} \frac{1}{2 n+4 m-3} \leq c_{n} \leq 2 \sum_{m=1}^{N} \frac{1}{2 n+4 m-3} .
$$

Therefore, we obtain by Lemma 6.1

$$
c_{n} \approx \sum_{m=1}^{N} \frac{1}{2 n+4 m-3} \approx \frac{1}{2 n+1}+\frac{1}{4} \ln \left(\frac{2 n+4 N-3}{2 n+1}\right) .
$$

From (6.4) we have

$$
\begin{aligned}
{\left[\frac{1}{2} \sum_{n=1}^{N-1} \frac{1}{n} c_{n}^{2}\right]-\left[\sum_{n=1}^{N-1} \frac{1}{n} b_{n}^{2}\right] } & \leq \sum_{n=1}^{N-1} \frac{1}{n}\left(\sum_{m=1}^{N} a_{m, n}\right)^{2} \\
& \leq 2\left[\sum_{n=1}^{N-1} \frac{1}{n} c_{n}^{2}\right]+2\left[\sum_{n=1}^{N-1} \frac{1}{n} b_{n}^{2}\right] .
\end{aligned}
$$

The estimates (6.5), (6.7) and Lemma 6.1 imply that

$$
\begin{aligned}
\sum_{n=1}^{N-1} \frac{1}{n} b_{n}^{2} & \lesssim \sum_{n=1}^{N-1} \frac{1}{n} \lesssim 1+\ln N, \\
\sum_{n=1}^{N-1} \frac{1}{n} c_{n}^{2} & \approx\left[\sum_{n=1}^{N-1} \frac{1}{n^{3}}\right]+\left[\sum_{n=1}^{N-1} \frac{1}{n} \ln ^{2}\left(\frac{2 n+4 N-3}{2 n+1}\right)\right] \\
& \approx 1+\sum_{n=1}^{N-1} \frac{1}{n} \ln ^{2}\left(\frac{2 n+1}{2 n+4 N-3}\right) .
\end{aligned}
$$

So it only remains to estimate $\sum_{n=1}^{N-1}(1 / n) \ln ^{2}[(2 n+1) /(2 n+4 N-3)]$. By Lemma 6.1 we have

$$
\begin{aligned}
\sum_{n=1}^{N-1} & \frac{1}{n} \ln ^{2}\left(\frac{2 n+1}{2 n+4 N-3}\right) \\
& \approx \sum_{n=1}^{N-1} \frac{24(N-1)}{(2 n+1)(2 n+4 N-3)} \ln ^{2}\left(\frac{2 n+1}{2 n+4 N-3}\right) \\
& \approx(1+\ln N)^{2}+\int_{1}^{N-1} \frac{24(N-1)}{(2 x+1)(2 x+4 N-3)} \ln ^{2}\left(\frac{2 x+1}{2 x+4 N-3}\right) d x \\
& =(1+\ln N)^{2}+\left[\ln ^{3}\left(\frac{2 x+1}{2 x+4 N-3}\right)\right]_{1}^{N-1} \\
& \approx(1+\ln N)^{3} .
\end{aligned}
$$

The lemma follows from (6.8)-(6.11).

Proof of Lemma 3.7. By the symmetry of $\hat{g}_{\rho}$, it suffices to estimate the first term $\left|\hat{g}_{\rho}-\hat{\Pi}_{\frac{1}{2}} \hat{g}_{\rho}\right|_{H_{00}^{1 / 2}(0,1 / 2)}$. According to Lemma 3.1 and (6.2), we have

$$
\left|G_{N}-\hat{\Pi}_{\frac{1}{2}} G_{N}\right|_{H_{00}^{1 / 2}(0,1 / 2)}^{2} \approx \sum_{n=1}^{\infty} \frac{1}{n}\left(\sum_{m=1}^{N} a_{m, n}\right)^{2} .
$$


Hence it follows from Lemmas 6.2-6.4 that

$$
\left|G_{N}-\hat{\Pi}_{\frac{1}{2}} G_{N}\right|_{H_{00}^{1 / 2}(0,1 / 2)}^{2} \approx(1+\ln N)^{3}=(1+|\ln \rho|)^{3} .
$$

On the other hand, as in the proof of Lemma 3.3, standard interpolation error estimates and (3.11) imply that

$$
\left|G_{N}-\hat{g}_{\rho}\right|_{H_{00}^{1 / 2}(0,1 / 2)} \lesssim 1
$$

Since $\hat{\Pi}_{\frac{1}{2}} G_{N}=\hat{\Pi}_{\frac{1}{2}} \hat{g}_{\rho}$, the estimate (3.24) follows from (6.12) and (6.13).

We now turn to the proof of Lemma 3.8. First we consider, for $N \geq 4$, the function $H_{N}$ on $[0,1 / 2]$ defined by

$$
H_{N}(x)= \begin{cases}G_{N}(x) & 0 \leq x \leq \frac{1}{2}-\frac{1}{N} \\ {\left[\frac{N}{2} G_{N}\left(\frac{1}{2}-\frac{1}{N}\right)\right](1-2 x)} & \frac{1}{2}-\frac{1}{N} \leq x \leq \frac{1}{2}\end{cases}
$$

Let $\sum_{n=1}^{\infty} \beta_{n} \sin (2 n \pi x)$ be the sine series of $H_{N}$ on the interval $(0,1 / 2)$. Using (3.10) and (6.14), we find

$$
\begin{aligned}
\beta_{n} & =4 \int_{0}^{1 / 2} H_{N}(x) \sin (2 n \pi x) d x \\
& =\frac{2}{n \pi} \int_{0}^{1 / 2} H_{N}^{\prime}(x) \cos (2 n \pi x) d x \\
& =\frac{2}{n \pi}\left[\pi \sum_{m=1}^{N} \int_{0}^{\frac{1}{2}-\frac{1}{N}} \cos ((4 m-3) \pi x) \cos (2 n \pi x) d x\right. \\
& \left.-N G_{N}\left(\frac{1}{2}-\frac{1}{N}\right) \int_{\frac{1}{2}-\frac{1}{N}}^{1 / 2} \cos (2 n \pi x) d x\right] .
\end{aligned}
$$

Note that

$$
\begin{aligned}
\int_{0}^{\frac{1}{2}-\frac{1}{N}} & \cos ((4 m-3) \pi x) \cos (2 n \pi x) d x \\
= & \frac{1}{2 \pi}\left[\frac{\sin ((2 n+4 m-3) \pi x)}{2 n+4 m-3}+\frac{\sin ((2 n-4 m+3) \pi x)}{2 n-4 m+3}\right]_{0}^{(1 / 2)-(1 / N)} \\
= & \frac{(-1)^{n}}{2 \pi}\left(a_{m, n}-b_{m, n}\right)
\end{aligned}
$$

where $a_{m, n}$ is defined by $(6.3)$ and

$$
b_{m, n}=\frac{1-\cos ((2 n+4 m-3) \pi / N)}{2 n+4 m-3}-\frac{1-\cos ((2 n-4 m+3) \pi / N)}{2 n-4 m+3} .
$$

Therefore $\beta_{n}$ can be written as

$$
\beta_{n}=(-1)^{n} \frac{2}{n \pi}\left[\frac{1}{2} \sum_{m=1}^{N}\left(a_{m, n}-b_{m, n}\right)-d_{n, N}\right]
$$

where

$$
d_{n, N}=N G_{N}\left(\frac{1}{2}-\frac{1}{N}\right) \frac{\sin (2 n \pi / N)}{2 n \pi}
$$


It is clear from (3.13) and (6.18) that

$$
\frac{1}{n} \sum_{n=N}^{\infty} d_{n, N}^{2} \lesssim N^{2} G_{N}\left(\frac{1}{2}-\frac{1}{N}\right)^{2} \sum_{n=N}^{\infty} \frac{1}{n^{3}} \lesssim(\ln N)^{2}
$$

On the other hand, using (3.10) and Lemma 6.1 we find

$$
G_{N}\left(\frac{1}{2}-\frac{1}{N}\right)=\sum_{n=1}^{N} \frac{1}{4 n-3} \cos \left(\frac{4 n-3}{N} \pi\right)=\frac{1}{4} \ln (4 N-3)+O(1) \quad \text { for } \quad n<N,
$$

and hence we obtain from (6.18)

$$
d_{n, N}=\frac{1}{4} \ln (4 N-3)+\frac{\ln N}{N^{2}} O\left(n^{2}\right)+O(1) \quad \text { for } \quad n<N .
$$

Lemma 6.5. It holds that $\sum_{n=1}^{\infty} \frac{1}{n}\left(\sum_{m=1}^{N} b_{m, n}\right)^{2} \lesssim \ln N$ for $N \geq 4$.

Proof. As in the proof of Lemma 6.2 , we have $\sum_{n=3 N+1}^{\infty} \frac{1}{n}\left(\sum_{m=1}^{N} b_{m, n}\right)^{2} \lesssim 1$.

On the other hand, in view of

$$
\begin{aligned}
& |1-\cos ((2 n+4 m-3) \pi / N)| \lesssim\left(\frac{2 n+4 m-3}{N}\right)^{2}, \\
& |1-\cos ((2 n-4 m+3) \pi / N)| \lesssim\left(\frac{2 n-4 m+3}{N}\right)^{2},
\end{aligned}
$$

which hold for $1 \leq n \leq 3 N$, we have $\left|\sum_{m=1}^{N} b_{m, n}\right| \lesssim 1$ for $1 \leq n \leq 3 N$, and therefore Lemma 6.1 implies that

$$
\sum_{n=1}^{3 N} \frac{1}{n}\left(\sum_{m=1}^{N} b_{m, n}\right)^{2} \lesssim \sum_{n=1}^{3 N} \frac{1}{n} \lesssim \ln N
$$

Lemma 6.6. It holds that $\left|H_{N}\right|_{H_{00}^{1 / 2}(0,1 / 2)}^{2} \approx(\ln N)^{3}$ for $N \geq 4$.

Proof. According to Lemma 3.1, we have $\left|H_{N}\right|_{H_{00}^{1 / 2}(0,1 / 2)}^{2} \approx \sum_{n=1}^{\infty} n \beta_{n}^{2}$. We deduce immediately from $(6.17),(6.19),(6.20)$ and Lemmas $6.2-6.5$ that

$$
\sum_{n=1}^{\infty} n \beta_{n}^{2} \lesssim(\ln N)^{3}
$$

To prove the reverse estimate, we observe from (6.6) and Lemma 6.1 that

$$
c_{n} \leq \frac{1}{2} \ln \left(\frac{2 n+4 N-3}{2 n+1}\right)+O\left(\frac{1}{n}\right) \quad \text { for } \quad n<N,
$$

which together with $(6.4),(6.5),(6.17)$ and (6.20) imply that

$$
\beta_{n}=(-1)^{n} \frac{2}{n \pi}\left(\gamma_{n}+\delta_{n}+\rho_{n}\right)
$$

where

$$
\gamma_{n} \geq \frac{1}{4} \ln (2 n+1), \quad \delta_{n}=\frac{\ln N}{N^{2}} O\left(n^{2}\right) \quad \text { and } \quad \rho_{n}=O(1) .
$$


Hence we have, by Lemma 6.1,

$$
\sum_{n=1}^{\infty} n \beta_{n}^{2} \geq \sum_{n=1}^{N-1} n \beta_{n}^{2} \geq \sum_{n=1}^{N-1} \frac{4}{n \pi^{2}}\left[\frac{\gamma_{n}^{2}}{2}-\left(\delta_{n}+\rho_{n}\right)^{2}\right] \gtrsim(\ln N)^{3}
$$

Lemma 6.7. It holds that $\left|\hat{\Pi}_{\rho} H_{N}\right|_{H_{00}^{1 / 2}(0,1 / 2)}^{2} \approx(\ln N)^{3}$ for $N \geq 4$.

Proof. We have, by (6.14),

$$
\left(H_{N}-\hat{\Pi}_{\rho} H_{N}\right)(x)= \begin{cases}\left(G_{N}-\hat{\Pi}_{\rho} G_{N}\right)(x) & 0 \leq x \leq \frac{1}{2}-\frac{1}{N}, \\ 0 & \frac{1}{2}-\frac{1}{N} \leq x \leq \frac{1}{2}\end{cases}
$$

Therefore, as in the proof of Lemma 3.3, standard interpolation error estimates and (3.11) imply that

$$
\left|H_{N}-\hat{\Pi}_{\rho} H_{N}\right|_{H_{00}^{1 / 2}(0,1 / 2)} \lesssim 1
$$

The lemma follows from (6.21) and Lemma 6.6.

Proof of Lemma 3.8. By the symmetry of $\hat{g}_{\rho}$, it suffices to estimate $\left|\hat{p}_{\rho}\right|_{H_{00}^{1 / 2}\left(0, \frac{1}{2}+\rho\right)}$.

For $N=2$, the estimate (3.27) is trivial. Let $N$ be greater than or equal to 4 , and $\hat{p}_{\rho}$ (resp. $\left.\hat{\Pi}_{\rho} H_{N}\right)$ be extended to be zero outside $\left(0, \frac{1}{2}+\rho\right)(\operatorname{resp} .(0,1 / 2))$. By $(3.25),(6.14)$ and recall $\hat{g}_{\rho}=\hat{\Pi}_{\rho} G_{N}$, we see that $\hat{p}_{\rho}-\hat{\Pi}_{\rho} H_{N}$ is a continuous function that vanishes outside $\left(\frac{1}{2}-\rho, \frac{1}{2}+\rho\right)$. Moreover, it is linear on $\left(\frac{1}{2}-\rho, \frac{1}{2}\right)$ and $\left(\frac{1}{2}, \frac{1}{2}+\rho\right)$, and equals $\frac{1}{2} G_{N}(1 / 2)$ at $x=1 / 2$. For such a function, a scaling argument yields

$$
\left|\hat{p}_{\rho}-\hat{\Pi}_{\rho} H_{N}\right|_{H^{1 / 2}(\mathbb{R})} \approx G_{N}(1 / 2) .
$$

The estimate (3.27) for $N \geq 4$ follows from (3.2), (3.13), (6.22), and Lemma 6.7.

\section{REFERENCES}

[1] J. Bergh and J. Löfström, Interpolation Spaces, Springer-Verlag, Berlin, 1976. MR 58:2349

[2] P. Bjørstad and J. Mandel, On the spectra of sums of orthogonal projections with applications to parallel computing, BIT, 31 (1991), 76-88. MR 91m:65157

[3] J.H. Bramble, J.E. Pasciak and A.H. Schatz, The construction of preconditioners for elliptic problems by substructuring, I, Math. Comp. 47 (1986), 103-134. MR 87m:65174

[4] S.C. Brenner and L.R. Scott, The Mathematical Theory of Finite Element Methods, SpringerVerlag, New York, 1994. MR 95f:65001

[5] T.F. Chan and T.P. Mathew, Domain decomposition algorithms Acta Numerica, 1994, pp. 61-143. MR 95f:65214

[6] P.G. Ciarlet, The Finite Element Method for Elliptic Problems, North Holland, Amsterdam, 1978. MR 58:25001

[7] M. Dryja, A method of domain decomposition for three dimensional finite element elliptic problems, First International Symposium on Domain Decomposition Methods for Partial Differential Equations (R. Glowinski, G.H. Golub, G.A. Meurant and J. Périaux, eds.), SIAM, Philadelphia, 1988, pp. 43-61. MR 90b:65200

[8] M. Dryja and O.B. Widlund, Some domain decomposition algorithms for elliptic problems, Iterative Methods for Large Linear Systems (L. Hayes and D. Kincaid, eds.), Academic Press, New York, 1989, pp. 273-291. CMP 90:07

[9] _ - Towards a unified theory of domain decomposition algorithms for elliptic problems, Third International Symposium on Domain Decomposition Methods for Partial Differential Equations (T. Chan, R. Glowinski, J. Pé riaux and O.B. Widlund, eds.), SIAM, Philadelphia, 1990, pp. 3-21. MR 91m:65294 
[10] _ Additive Schwarz methods for elliptic finite element problems in three dimensions, Fifth International Symposium on Domain Decomposition Methods for Partial Differential Equations (T.F. Chan, G.Meurant, J.S. Scroggs and R.G. Voigt, eds.), SIAM, Philadelphia, 1992, pp. 3-18. MR 93j:65201

[11] _ Schwarz methods of Neumann-Neumann type for three dimensional elliptic finite element problems, Comm. Pure Appl. Math. 48 (1995), 121-155. MR 96d:65199

[12] M. Griebel and P. Oswald, On the abstract theory of additive and multiplicative Schwarz algorithms, Numer. Math. 70 (1995), 163-180. MR 96a:65164

[13] F. Kickinger, S.V. Nepomnyaschikh, R. Pfau and J. Schöberl, Numerical Estimates of Inequalities in $H^{1 / 2}$, Technical Report No 97-3, Institut für Mathematik, Johannes Kepler Universität, Linz, 1997.

[14] S.G. Kreǐn, J. I. Petunin and E.M. Semenov, Interpolation of Linear Operators, Translations of Mathematical Monographs, Volume 54, American Mathematical Society, Providence, 1982. MR 84j:46103

[15] Y. Kuznetsov, P. Manninen and Y. Vassilevski, On numerical experiments with NeumannNeumann and Neumann-Dirichlet domain decomposition preconditioners, Technical Report, University of Jyväkylä, 1993.

[16] P. Le Tallec, Domain decomposition methods in computational mechanics, Comput. Mech. Adv. 1 (1994), 121-220. MR 95b:65147

[17] P. Lions, On the Schwarz alternating method. I, First International Symposium on Domain Decomposition Methods for Partial Differential Equations (R. Glowinski, G.H. Golub, G.A. Meurant and J. Périaux, eds.), SIAM, Philadelphia, 1988, pp. 1-42. MR 90a:65248

[18] J.L. Lions and E. Magenes, Non-Homogeneous Boundary Value Problems and Applications I, Springer-Verlag, Berlin, 1972. MR 56:2670

[19] A.M. Matsokin and S.V. Nepomnyaschikh, Schwarz alternating method in subspaces, Soviet Mathematics 29 (1985), 78-84.

[20] S.V. Nepomnyaschikh, On the application of the bordering method to the mixed boundary value problem for elliptic equations and on mesh norms in $W^{1 / 2}(S)$, Soviet J. Numer. Anal. Math. Modelling 4 (1989), 493-506.

[21] — Fictitious components and subdomain alternating methods, Soviet J. Numer. Anal. Math. Modelling 5 (1990), 53-68.

[22] B. Smith, P. Bjørstad and W. Gropp, Domain Decomposition, Cambridge University Press, Cambridge, 1996. MR 98g:65003

[23] H. Triebel, Interpolation Theory, Function Spaces, Differential Operators, North-Holland, Amsterdam, 1978. MR 80i:46032b

[24] X. Zhang, Studies in Domain Decomposition: Multi-level Methods and the Biharmonic Dirichlet Problem, Dissertation, Courant Institute, 1991.

[25] —, Multilevel Schwarz methods, Numer. Math. 63 (1992) 521-539. MR 93h:65047

Department of Mathematics, University of South Carolina, Columbia, SC 29208

E-mail address: brenner@math.sc.edu

Department of Mathematics, University of South Carolina, Columbia, SC 29208

E-mail address: sung@math.sc.edu 